\title{
Non-painful sensory phenomena after spinal cord injury
}

Philip J Siddall, Joan McClelland

\begin{abstract}
Objectives-Non-painful sensory phenomena or "phantom" sensations are common after spinal cord injury. However, the physiological mechanisms responsible for these sensations are poorly understood. The aim of this study, therefore, was to document in a prospective fashion the time course, prevalence, and features of non-painful sensory phenomena after spinal cord injury, and to determine whether there was a relation between the presence of these sensations and completeness, level of injury, and type of spinal cord injury.
\end{abstract}

Methods-Patients admitted to an acute spinal injuries unit were interviewed after admission and at several time points over a 2 year period to determine the presence and characteristics of non-painful sensations. Sensations were divided into simple and complex, with complex referring to sensations that incorporated a sensation of volume, length, posture, or movement. Results-The present study showed that the large majority $(90 \%)$ of patients experience either type of sensation and most complex sensations $(60 \%)$ are first experienced within 24 hours after the injury. Complex sensations were more common in those patients who had complete spinal cord injuries. The presence of either type of sensation did not seem to be related to the level of injury or the type of injury (cord syndrome). A relatively small proportion $(22 \%)$ of patients reported that the postural sensations were related to their position at the time of injury and sensations were more commonly related to a familiar, comfortable, or often used position before the spinal cord injury.

Conclusion-Complex sensations such as postural illusions seem to be due to functional changes in the CNS that may occur almost immediately after spinal cord injury. These sensations may be related to a strong sensory memory "imprint" that has been established before injury.

(F Neurol Neurosurg Psychiatry 1999;66:617-622)

Keywords: spinal cord injury; phantom; sensation

Management Centre, Royal

North Shore Hospital, St

Leonards, NSW, Australia

2065. Telephone 6129926

6387; fax 6129926 6548;

email

phils@med.usyd.edu.au

Received 2 April 1998 and in revised form

13 November 1998

Accepted 26 November 1998 as "phantom" sensations. These have been described after loss of parts of the body including limbs, ${ }^{2}$ breast $^{3}$ nose, ${ }^{1}$ and teeth ${ }^{4}$ and have been reported after the loss of other sensations such as vision. ${ }^{5}$ Phantom sensations may take the form of "simple" sensations such as pressure, temperature and touch, and more "complex" sensations such as posture, length, and volume. ${ }^{6}$ There may also be sensations of movement such as spontaneous or willed movements of the affected region.

Many patients who have sustained a spinal cord injury also report sensory phenomena that have many similarities to these phantom limb sensations that are experienced after amputation. ${ }^{17-11}$ After spinal cord injury, some patients describe an "amputation illusion" or a sensation that the anaesthetic part is no longer present. ${ }^{1811}$ This absence of sensation is often replaced by the presence of sensations which are perceived as originating from the region of sensory disturbance. ${ }^{8}{ }^{12}$ These sensations may include a feeling of tightness, heaviness, burning, coldness, tingling, vibration, numbness, formication, and swelling. The limbs may be perceived to be in a posture which is unrelated to their real position and the lower limbs are commonly described as being flexed at the hip and knee joint, lifted into the air, or crossed. ${ }^{8912}$ Sensations of complex movements such as cycling of the legs ${ }^{9}$ or sensations of reflex actions such as penile erection and orgasm may also occur. ${ }^{10}$

Although these non-painful sensory phenomena after spinal cord injury have been described, there is little information available about the prevalence and duration of these sensations or about their relation with other variables associated with the injury..$^{90}{ }^{13-14}$ It has been suggested that the prevalence of phantom sensations after traumatic spinal cord injury is as high as $89 \%{ }^{10}$ or $100 \%{ }^{8}$ It has also been reported that the sensations tend to disappear with time. ${ }^{8}$ However, the information that has been obtained in these studies has usually been collected some time after injury. The retrospective nature of these studies, therefore, makes it difficult to determine accurately the time of onset and duration of phantom sensations.

The relation of phantom sensations to physical factors associated with the spinal cord injury also seems uncertain. It has been reported that the presence of phantom sensations is related to the spinal cord level of injury or age ${ }^{89}$ It is also commonly stated that the perceived position of the limbs is related to their position at the time of injury. ${ }^{12}{ }^{14}$ Others have failed to find this relation. ${ }^{9}$ 
Thus, the presence of non-painful sensations in the region of sensory disturbance is a well recognised phenomenon after spinal cord injury. However, there are no prospective studies which have documented the characteristics of these sensations from the time of injury. The aim of this study, therefore, was to determine prospectively the nature, onset, and time course of phantom sensations after spinal cord injury. Furthermore, we sought to determine whether the presence of these sensations was related to other factors such as level of injury, completeness, or type of spinal cord injury, and whether the nature of these sensations was related to factors such as position at the time of injury.

\section{Methods}

PATIENTS

We performed a prospective, longitudinal study of patients admitted to the spinal injuries unit of this institution with acute spinal cord injury. This institution admits about $60 \%$ of all people in the region (population 6.2 million) who have spinal cord injuries. Selection for admission is based on geographical location at the time of injury. The study protocol was approved by the institutional human ethics review committee. All people admitted to the unit within 3 months of an acute traumatic spinal cord injury were asked to participate in the study. People were excluded if they were under 18 years of age, had a history of psychiatric disorder, sustained a brain injury during their accident which resulted in continuing neuropsychological deficit, or had difficulty with communication. Communication difficulties included confusion and lack of fluency in the English language. People who were being ventilated during the course of the study were also not included. If possible, participants gave informed written consent. Those who were unable to write gave verbal consent. Results obtained from patients who were discharged with no sensory or motor deficit were not included in the analysis.

Of the 106 patients who were eligible for inclusion in the study, 103 agreed to partici-

Table 1 Patient characteristics

\begin{tabular}{lc}
\hline Item & No (total $n=103)$ \\
\hline Total & 103 \\
Male & 84 \\
Mean age (range) & $38(18-78)$ \\
Tetraplegic & 52 \\
Aetiology: & \\
Motor accident & 43 \\
Fall & 25 \\
Sporting accident & 19 \\
Bicycle & 5 \\
Crush injury & 6 \\
Miscellaneous & 5 \\
Level (neurological): & \\
Cervical & 54 \\
Thoracic & 26 \\
Lumbar & 22 \\
Sacral & 1 \\
Syndrome: & 19 \\
Central cord & 6 \\
Anterior cord & 9 \\
Brown-Sequard & 12 \\
Conus medullaris & 15 \\
Cauda equina & 38 \\
Complete & \\
\hline
\end{tabular}

pate. Characteristics of the patients who took part in the study are shown in table 1. People were interviewed as soon as possible after their injury. This usually occurred 14 days after their injury. Interviews occurred at 2 week intervals until discharge from the spinal injuries unit and at 3, 6, 12, and 24 months after injury. Some patients were not available for interview at some of these times ( 1 month, $\mathrm{n}=103 ; 12$ months, $n=72 ; 24$ months, $n=44)$. This was due to the death of two patients and loss to follow up because of relocation after discharge.

Patients were interviewed by a researcher in the study with our own questionnaire, which recorded the presence and features of sensations in areas of sensory disturbance. These were divided into painful and non-painful. Only non-painful sensations that occurred in the region of sensory disturbance at or below the level of injury were included in this study. Non-painful sensations were classified into two groups: simple and complex. ${ }^{6}$ Simple sensations refer to non-painful sensations other than position or movement such as tightness, heaviness, tingling, vibrating, numbness, formication and swelling. Complex sensations refer to sensations that may have the same qualities as simple sensations but also include a sensation of length, volume, posture, or movement. These include sensations such as positional illusions of the limbs, limb movement and functional illusions such as defaecation or micturition. People were not informed of prior responses.

Variables of interest related to the spinal cord injury such as cause and type of injury, and level and completeness of lesion were also recorded. Confounding variables such as medication intake were also recorded. Completeness was judged on the basis of physical examination and the term was used when there was absence of sensory and motor function in the lowest sacral segment. ${ }^{15}$

DATA ANALYSIS

$\chi^{2}$ Tests with Yates' correction and Fisher's exact test were used to compare groups. Significance was defined as $\mathrm{p}<0.05$.

\section{Results}

ONSET

Simple sensations

The average time of onset of simple sensations was 75.8 (SD 126.1) days after injury. Twenty seven per cent of simple sensations started within 24 hours of injury; $23 \%$ started after the first day but within the first week; $22 \%$ started after the first week but within the first month; $28 \%$ started more than 1 month after injury (fig 1).

\section{Complex sensations}

By contrast with simple sensations, the onset of complex sensations was generally earlier. The average time of onset was $8.3 \mathrm{~d}$ (SD 20.6) days after injury. Sixty per cent of complex sensations started within 24 hours of injury; $20 \%$ started after the first day but within the first week; $12 \%$ commenced after the first week but 


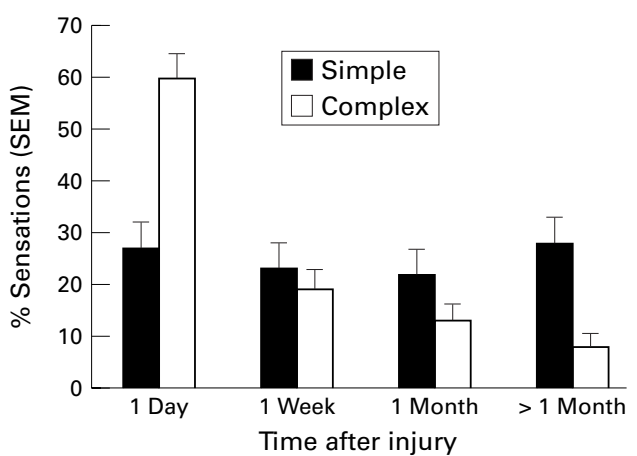

Figure 1 Percentage of simple and complex sensations which appeared for the first time at different time points after spinal cord injury.

within the first month; $8 \%$ started more than 1 month after injury (fig 1).

INCIDENCE AND PREVALENCE

Of the 73 patients who were assessed for the first 12 months after their injury, 66 (90\%) had non-painful sensations in the region of sensory disturbance at some time during this time (table 2). Forty seven patients $(63 \%)$ had simple sensations; $45(62 \%)$ had complex sensations; 13 (18\%) experienced both types of sensations and some patients had more than one sensation of the same type. In total, there were 236 (120 simple; 116 complex) sensations reported among 73 patients.

The percentage of patients who reported either simple or complex sensations remained similar over the 2 year period and did not fall below $50 \%$ (fig 2). However, the prevalence of the two types of sensations displayed different patterns. Simple sensations were present in $21 \%$ of patients at day 1 after injury but 1 year after injury, $47 \%$ of patients reported simple sensations. By contrast, complex sensations were present in $54 \%$ of patients at day 1 but this had decreased to $24 \%$ at 2 years.

LOCATION OF SENSATIONS

The majority of both simple and complex sensations $(59 \%$ and $62 \%$ respectively) were located in the lower limbs (table 3). The next most common location was in the upper limbs. Sensations were perceived to arise from the upper limbs in $21 \%$ of those with simple sensations and $19 \%$ of those with complex sensations. In $17 \%$ of those with complex sensations, these were perceived to arise from the bladder, bowels, or genitalia or were associated with bladder or bowel function.

\section{DESCRIPTION}

Simple sensations

Various descriptors were used to characterise simple sensations. These descriptors included sensations of light touch, tightness, itching, bursting, pressure, temperature change (cold

Table 2 Incidence of non-painful sensations (at 1 year)

\begin{tabular}{lllll}
\hline & Simple & Complex & Either & Both $^{\star}$ \\
\hline $\begin{array}{l}\text { No of patients } \\
\text { \% Of study population }\end{array}$ & 47 & 45 & 66 & 13 \\
\hline
\end{tabular}

${ }^{\star}$ Some patients had both simple and complex sensations.

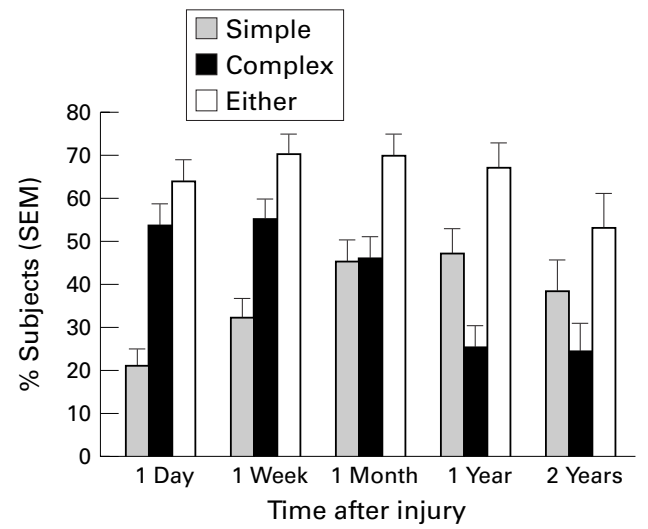

Figure 2 Percentage of patients with different types of sensations at different time points after spinal cord injury. (Numbers of patients in each group: 1 day, $n=103 ; 1$ week, $n=103 ; 1$ month, $n=103 ; 1$ year, $n=72 ; 2$ years, $n=44$.)

or hot), cramping, restlessness, brushing, throbbing, heaviness, tingling, cramping, cutting, prickling, fuzziness, and electricity. Those most commonly reported were tingling (13 patients) and pins and needles (10 patients). Each of the other sensations were reported by between one and five patients.

\section{Complex sensations}

The greatest number of complex sensory disturbances that were reported by patients were positional sensations perceived to arise from the lower limbs. Most patients described their lower limbs in either a position of hip and knee flexion or hip adduction (table 4). Thirty five were asked if they could voluntarily "move" the perceived position of their phantom limbs and they could not. Three out of 10 (30\%) found that the phantom disappeared when they visually checked the position of their limbs. Fifteen were asked about the presence of shortening or telescoping. These terms refer to the sensation of the end of the limb being closer to the torso and therefore a perception that the limb had shortened. None of the patients in this study experienced these phenomena. Nineteen were asked about a sensation of "amputation" of the region of sensory loss. Three of them (16\%) experienced this.

Nine patients reported sensations that also involved movement. Four of the nine reported that the legs felt as though they were swinging. The other reports included a sensation of movement in the hands and fingers, including one in which there was a sensation of picking up something between the fingers. Sixteen patients reported sensations associated with micturition (eight patients) or defaecation (eight patients).

Several patients reported sensations which were clearly related to prior experiences. One reported a sensation like a band around her finger. This was identical to the sensation she used to experience when putting an elastic band around her finger while brushing her daughter's hair. One patient reported a feeling of dislocation of her knee identical to that experienced some years previously except that it was no longer painful. One patient reported a cramping sensation in the right calf. This sen- 
Table 3 Location (n (\%)) of non-painful sensations

\begin{tabular}{llllllll}
\hline & $\begin{array}{l}\text { Upper } \\
\text { limbs }\end{array}$ & Torso & $\begin{array}{l}\text { Lower } \\
\text { limbs }\end{array}$ & $\begin{array}{l}\text { Rectum } \\
\text { bowels }\end{array}$ & $\begin{array}{l}\text { Bladder } \\
\text { urination }\end{array}$ & Genitalia & $\begin{array}{l}\text { Whole } \\
\text { body }\end{array}$ \\
\hline Simple & $29(21)$ & $13(9)$ & $81(59)$ & $5(4)$ & $0(0)$ & $1(1)$ & $9(7)$ \\
Complex & $22(19)$ & $1(1)$ & $71(62)$ & $8(7)$ & $8(7)$ & $3(3)$ & $2(2)$ \\
\hline
\end{tabular}

Table 4 Types of positional sensations

\begin{tabular}{ll}
\hline Position & $\begin{array}{c}\text { No of patients } \\
\text { (total } n=103 \text { ) }\end{array}$ \\
\hline Lower limbs: & \\
Hips flexed, knees flexed & 19 \\
Legs crossed & 16 \\
Legs, feet externally rotated & 7 \\
Knees flexed & 5 \\
Feet hanging over edge of bed & 4 \\
Legs spread apart & 3 \\
Feet plantar flexed & 3 \\
Heels pressing into buttocks & 3 \\
Toes curling & 2 \\
Toes pressing together & 1 \\
Legs twisted around each other & 1 \\
Legs up over shoulders & 1 \\
Foot twisting & 1 \\
Ankles rubbing together & 1 \\
Upper limbs: & \\
Hands clenched & 6 \\
Arms extended & 3 \\
Arms and hands clasped in lap & 1 \\
Arms crossed over body & 1 \\
Fingers flexed & 1 \\
Hand overlapping & 1 \\
Right thumb pulled back & 1 \\
Left hand upside down & 1 \\
Arms behind back & 1 \\
Arms twisted around throat & 1 \\
Hands on abdomen & 1 \\
Left arm abducted & 1 \\
Body/torso: & 1 \\
Disconnected, floating & \\
Pelvis rotated 90 & \\
Body turned to side & \\
Back arching & \\
Body tilted (feet down) & \\
\hline & \\
\hline
\end{tabular}

sation was identical to that experienced on the morning of the accident which resulted in his spinal cord injury when he had experienced difficulties in starting his motor cycle.

PRODUCTION OF CHANGES IN SENSATIONS

Simple sensations

Various situations or factors seemed to induce simple sensations. These are listed in table 5 .

Complex sensations

Several situations produced changes in complex sensations. One patient reported that positional sensations disappeared temporarily after administration of diazepam. One reported that approaching doorways would produce a sensation that the left arm was abducted. One reported that the sensations did not start until immediately after spinal surgery. One reported that the sensations ceased when he was rolled into the supine position and did not return. One reported a sensation of uncontrolled bowel actions. This started when he moved

Table 5 Simple sensation triggers *

\begin{tabular}{ll}
\hline Trigger & Effect \\
\hline Focusing on moving a part & Tingling (two patients) \\
Smoking & Burning or tingling (two patients) \\
Abdominal spasm & Electric sensations \\
Urinary tract infection & Abdominal tightness \\
Sudden noise & Electric sensations \\
\hline
\end{tabular}

^All patients had complete spinal cord lesions.

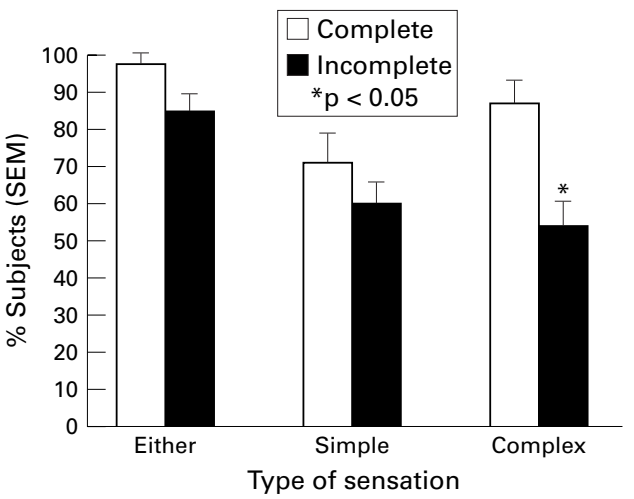

Figure 3 Percentage of patients with different types of sensations according to completeness of the spinal cord injury. (Numbers of patients in each group: complete, $n=38$; incomplete, $n=65$.)

into a room in which another person had had frequent bowel accidents.

\section{RELATION TO OTHER VARIABLES}

There was a significantly higher proportion of patients with complex sensations in the group that had complete injuries $(87 \%)$ compared with the group that had incomplete injuries (54\%) (Yates' corrected $\chi^{2}=10.21, \mathrm{df}=1$, $\mathrm{p}=0.0014$ ) (fig 3). There was no significant difference in the proportion of patients with non-painful sensations of either type or nonpainful simple sensations when those who had complete spinal cord injuries were compared with those who had incomplete injuries.

There was no significant difference in the proportion of patients reporting simple, complex, or either sensation when comparisons were made of patients with injuries at cervical, thoracic, and lumbar spinal levels (fig 4).

Eighty three per cent of those with conus medullaris experienced complex sensations compared with $42 \%$ and $47 \%$ with central cord and cauda equina injuries respectively. However, these proportions failed to reach significance (fig 5).

Of those who could remember their position at the time of injury, $12 / 55(22 \%)$ reported that their positional sensations were identical to those experienced at the time of injury. Fifteen out of $38(39 \%)$ reported that they were identical to a familiar, comfortable, or often used

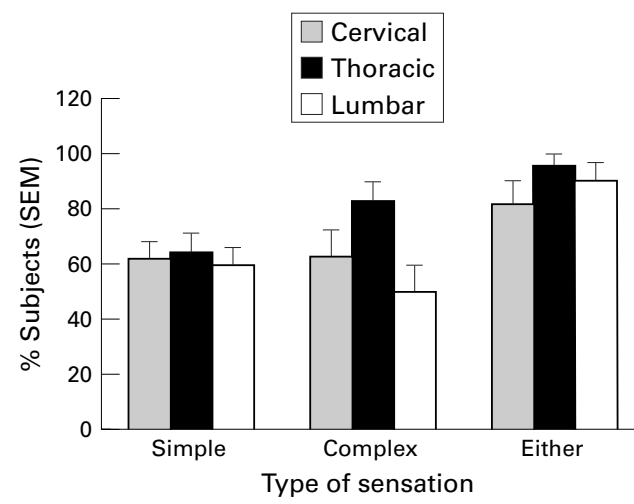

Figure 4 Percentage of patients with different types of sensations according to the level of spinal cord injury. (Numbers of patients in each group: cervical, $n=54$; thoracic, $n=26$; lumbar, $n=22$.) 


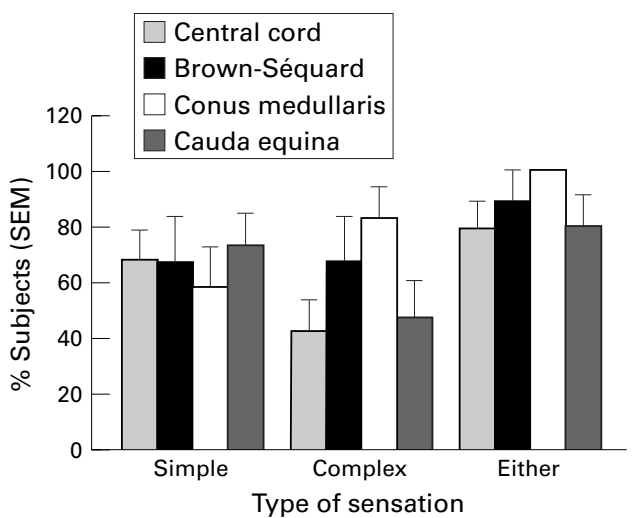

Figure 5 Percentage of patients with different types of sensations according to type of spinal cord injury. (Numbers of patients in each group: central cord, $n=19$;

Brown-Sequard, $n=9$; conus medullaris, $n=12$; cauda equina, $n=15$.)

position before their injury. None of the simple sensations were the same as those experienced at the time of injury. Eleven out of $28(39 \%)$ simple sensations were also identical to familiar, comfortable, or often experienced sensations before injury. Two out of the four $(50 \%)$ movement sensations were familiar, comfortable, or movements experienced before injury. None of the movement sensations seemed to be related to the injury experience. There was no relation between the presence of pain (neuropathic below level) and non-painful sensations.

\section{Conclusions}

This is the first prospective study which documents the prevalence, time course, and features of non-painful sensory phenomena after spinal cord injury from the time of injury. This study shows that there are two main types of sensory phenomena that differ in their time course and are related to different variables after spinal cord injury. Specifically, the study shows that those patients with complete spinal cord injury lesions were more likely to report complex sensations than those with incomplete lesions. However, there was no relation between injury level or type of injury and the presence of either simple or complex sensations after spinal cord injury.

There are several factors in a study of this type that may distort the findings and conclusions. Selection bias, loss of patients to follow up, and confounding variables during the course of the study may all contribute to inaccuracies. Attempts were made to keep these factors to a minimum. Selection bias was reduced because admission to the hospital was based purely on the site where injury occurred and was not related to factors associated with the injury or the patient. The high number of eligible participating patients (97\%) also reduced bias related to those who entered in the study. It is acknowledged that report of sensations may have been affected by medication and other intervention such as surgery. These variables were noted but no consistent relation between either of these factors and a change in sensations was seen.
The prevalence of sensations after spinal cord injury is within the range of figures obtained in previous studies. However, the range is quite large with previous studies reporting that $51 \%,{ }^{14} 89 \%,{ }^{10} 94 \%,{ }^{9}$ and $100 \%{ }^{8}$ of patients experience phantom sensations after their injury. One reason for the large range of percentages may be the definition of phantom sensations that we used. The study reporting the lowest percentage ${ }^{14}(51 \%)$ only includes those with positional illusions whereas those with higher percentages include other types of sensory phenomena including pain. ${ }^{8-10}$ In the present study, we included all nonpainful sensory phenomena but did not include pain and therefore the percentage that we found $(90 \%)$ is at the lower end of those studies which included both pain and other sensations.

By contrast with previous reports, we found that most patients $(60 \%)$ who experience complex sensations after spinal cord injury first report them within 24 hours after the injury. ${ }^{16}$ On the other hand, simple sensations tended to appear later. Previous reports have suggested that spinal cord injury "phantoms" tend to appear later than amputation "phantoms", which occur immediately or soon after amputation. ${ }^{2}$ The reason for this difference in the present study may be that previous studies of sensory phenomena after spinal cord injury are retrospective and that patients find it difficult to accurately recall events immediately after their injury.

The difference in time course for simple sensations also suggests that different mechanisms may be responsible for the presence of simple and complex sensations. It has been suggested that sprouting, which has been seen to occur after peripheral nerve damage, may be responsible for the appearance of abnormal sensory phenomena after peripheral nerve injury. ${ }^{17}{ }^{18}$ Although this may be the case with simple sensations, the short time course of complex sensations after spinal cord injury (majority within 24 hours after injury) seems to be inconsistent with the phenomenon of sprouting, which does not occur for several days after the injury. ${ }^{18}$

One feature of phantom sensations that is seen after limb amputation is the phenomenon of "telescoping" or apparent shortening of the phantom limb. ${ }^{26}$ In agreement with the findings of Bors ${ }^{8}$ and Conomy, ${ }^{9}$ none of the patients in the present study experienced shortening or telescoping of the phantom. It has also been reported that patients with spinal cord injury experience an amputation illusion immediately after injury. ${ }^{10} 11$ In accord with these findings, $16 \%$ of those questioned in the present study experienced this sensation.

Of interest in any study of this nature is what can be learned of the mechanisms responsible for the generation of the sensations. Although it is easier to hypothesise mechanisms that may result in the perception of simple sensations, the mechanisms responsible for complex sensations which may include a sensation of position or even movement are more difficult to understand. It has been suggested that the 
position of the limbs, as perceived by the patient, is related to position at the time of injury. ${ }^{10} 1214$ The findings from our own study indicate that some patients do have phantom sensations that are related to the time of injury. However, there was a relatively small proportion $(22 \%)$ of patients who had complex sensations corresponding to their injury position in the present study. This finding disputes the view suggested by some authors ${ }^{12}{ }^{14}$ that this association is an almost universal phenomenon. The findings of the present study are supported by Conomy ${ }^{9}$ who found only a few patients in which this was the case. It is interesting that most patients in the present study reported sensations of either lower limb flexion or adduction and rarely extension. This is in agreement with the results of Riddoch ${ }^{1}$ and Conomy ${ }^{9}$ who noted that the limbs are often initially perceived to be in a position of flexion, even when the patient was in a position of extension at the time of injury.

Some patients report positional illusions that are identical to positions that are often used, comfortable, or familiar before their injury. In an extensive review of the subject Sweet ${ }^{11}$ mentions a case described by Mayer-Gross ${ }^{19}$ in which the patient with spinal cord injury had a phantom sensation in which the forearms were folded and hands clasped in a favourite position for him and not the position at the time of injury. In our study, one woman had a sensation which was identical to that experienced when she used to tie an elastic head band around her finger to do her daughter's hair some years previously. Many patients reported that the position of the phantom in which they were lying down with the hips and knees flexed and feet on the bed was a comfortable or often used position.

The association between preinjury positions (either past or immediately before) leads to the postulation of mechanisms which are largely conjectural. It has been suggested that they are due to: (1) the creation of "artificial synapses"13; (2) preservation of afferent input"; (3) the result of a "learned" or inbuilt memory or "body schema" or "neuromatrix"20-22; and (4) Loss of afferent input and memory "gridlock". ${ }^{14}$ Our findings, as well as those of others, supports the concept that spinal cord injury sensory phenomena are related to prior sensations that are linked to repetitive events. This supports the idea of "imprinted" sensory memories. We suggest that phantom sensations are related to any input which occurs at any stage before spinal cord injury, which is either strong or repetitive and therefore has a strong "imprint" in long term memory.

There is another feature of non-painful sensations that may also provide clues as to underlying mechanisms. They are often triggered by input from other sources. One woman in the present study developed a feeling that her knee was dislocated 12 weeks after the injury although it was not regarded as painful. This was triggered by a urinary tract infection. It has been previously been reported that phantom sensations are triggered by stimulation of areas remote to the site of the sensation. It has been noted in studies of other phantom phenomena, such as phantom breast sensation, ${ }^{23}$ that perceptual remapping occurs after loss of sensory input. Thus, sensory input from a region with preserved sensory function after spinal cord injury may be responsible for the perception of sensations in a remote region that no longer provides sensory input.

We thank Professor Michael Cousins, David Taylor, and Kathy Gustafson for their helpful suggestions, advice, and support during the study. We thank Dr Fiona Blyth for her constructive comments on the manuscript and Jack Chen for statistical advice. We also acknowledge and appreciate the ongoing support and input from Dr Sue Rutkowski and Dr James Middleton of the Royal North Shore Hospital Spinal Injuries Unit, Sydney, Australia. This project was supported by WorkCover's Injury Prevention, Education and Research Grants Scheme, and the Motor Accidents Authority, NSW, Australia.

1 Riddoch G. Phantom limbs and body shape. Brain 1941;64:197-222

2 Jensen TS, Krebs B, Nielsen J, et al. Non-painful phantom limb phenomena in amputees: incidence, clinical characteristics and temporal course. Acta Neurol Scand 1984;70 407-14.

3 Kroner K, Knudsen UB, Lundby L, et al. Long-term phantom breast syndrome after mastectomy. Clin F Pain 1992;8: 346-50.

4 Pollman L. Phantom pain also following tooth extraction. Fortshcr Med 1990;108:59-61.

5 Schultz G, Melzack R. Visual hallucinations and mental Schultz G, Melzack R. Visual hallucinations and mental
state - a study of 14 Charles Bonnet syndrome hallucinastate - a study of 14 Charles Bonnet syndr
tors. F Nerv Ment Dis 1993;181:639-43.

tors. F Nerv Ment Dis 1993;181:639-43.
6 Jensen TS, Rasmussen P. Phantom pain and other phenomJensen TS, Rasmussen P. Phantom pain and other phenom-
ena after amputation. In: Wall PD, Melzack R, eds. Textbook of pain. Edinburgh: Churchill Livingstone, 1994:651-65.

7 Riddoch G. The reflex functions of the completely divided spinal cord in man, compared with those associated with less severe lesions. Brain 1917;40:264-402.

8 Bors E. Phantom limbs of patients with spinal cord injury. American Medical Association Archives of Neurology and Psychiatry 1951;66:610-31.

9 Conomy JP. Disorders of body image after spinal cord injury. Neurology 1973;23:842-50.

10 Berger M, Gerstenbrand F. Phantom illusions in spinal cord lesions. In: Siegfried J, et al, eds. Phantom and stump pain. Berlin: Springer-Verlag, 1981:66-73.

11 Sweet WH. Phantom sensations following intraspinal injury. Neurochirurgia Stuttgart 1975;18:139-54.

12 Bedbrook GM. Pain and phantom sensation. In: Bedbrook $\mathrm{GM}$, ed. The care and management of spinal cord injuries. GM, ed. The care and management of

13 Abram SE, Yaksh TL. Morphine, but not inhalational anesthesia, blocks post-injury facilitation. Anesthesiology 1993;78:713-21.

14 Ettlin TM, Seiler W, Kaeser HE. Phantom and amputation illusions in paraplegic patients. Eur Neurol 1980;19:12-19. 5 Waters RL, Adkins RH, Yakura JS. Definition of complete spinal cord injury. Paraplegia 1991;9:573-81.

16 Wall PD. On the origin of pain associated with amputation. In: Phantom and stump pain. Berlin: Springer-Verlag, 1981: $2-14$.

17 Woolf C, Shortland P, Coggeshall RE. Peripheral nerve injury triggers central sprouting of myelinated afferents. Nature 1992;355:75-8.

18 Woolf CJ, Shortland P, Reynolds M, et al. Reorganization of central terminals of myelinated primary afferents in the rat dorsal horn following peripheral axotomy. f Comp Neurol dorsal horn followin

19 Mayer-Gross W. Ein Fall von Phantomarm nach Plexuszerreissung. Nervenarzt 1929;2:65-72.

20 Melzack R, Loeser JD. Phantom body pain in paraplegics: evidence for a central "pattern generating mechanism" for pain. Pain 1978;4:195-210.

21 Melzack R. Phantom limbs. Sci Am 1992;266:120-6.

2 Melzack R. Phantom limbs and the concept of a neuromatrix. Trends Neurosci 1990;13:88-92.

23 Aglioti S, Cortese F, Franchini C. Rapid sensory remapping in the adult human brain as inferred from phantom breast perception. Neuroreport 1994;5:473-6. 